\title{
Metabolic resuscitation therapy in critically III patients with sepsis and septic shock: A Prospective Randomized Controlled Trial
}

\section{Fang Feng}

Lanzhou University Second Hospital

Huyong Yang

People`s Hospital of Linxia State

\section{Weiwei Yang}

People`s Hospital of Linxia State

\section{Min Li}

Lanzhou University Second Hospital

Yu Chen ( $\square$ chenyu14@Izu.edu.cn )

Lanzhou University Second Hospital https://orcid.org/0000-0003-1447-4410

\section{Research}

Keywords: Metabolic resuscitation, Sepsis, Septic shock

Posted Date: January 8th, 2021

DOl: https://doi.org/10.21203/rs.3.rs-140461/v1

License: (c) (1) This work is licensed under a Creative Commons Attribution 4.0 International License. Read Full License 


\section{Abstract}

Objective

To further clarify the effectiveness and potential pathophysiological principles of metabolic resuscitation therapy in critically ill patients with sepsis and septic shock.

Methods

Prospective randomized controlled trial. Patients with sepsis and septic shock who were admitted to the ICU from Sep. 2019 to Mar. 2020 were prospectively enrolled. According to the computerized random sequence table, patients were randomly divided into the experimental group and the control group. All patients with sepsis were included in the research of cluster of initial therapy. The experimental group received metabolic resuscitation: vitamin $\mathrm{C}$ ( $1.5 \mathrm{~g}$ in an intravenous infusion q6h for 3 days), vitamin B1 (200 $\mathrm{mg}$ in an intramuscular injection q12h for 3 days), and hydrocortisone (50 $\mathrm{mg}$ in an intravenous infusion q6h for 7 days).

Results

One hundred thirty-six patients with sepsis and septic shock were included in our study. Pneumonia was the main cause of sepsis and septic shock, including $33(49 \%)$ and $41(60 \%)$ patients in the experimental group and control group, respectively, and gram-negative bacilli were the main pathogenic bacteria $(112 / 136,82.4 \%)$. The ICU length of stay for the experimental group and the control group was $9(7-12)$ and 11 (9-14) days, respectively, $P=0.002$. The duration of vasoactive drugs (hours) was $20.8 \pm 9.9$ and $46.7 \pm 12.8$, respectively, $\mathrm{P} \otimes 0.001$. The 6 -hour lactate clearance rate was $66.2 \%(55.5,76.7)$ and $30.1 \%$ $(15.6,50)$ in the experimental group and control group, respectively, $P=0.000$. The 72 -hour PCT clearance rate was $70 \%(57.5,80.3)$ and $40.7 \%(24.8,52.2)$, respectively, $P=0.001$. The ICU mortality rate was $8.8 \%$ $(6 / 68)$ and $15 \%(15 / 68)$, respectively, $P=0.033$.

\section{Conclusions}

Metabolic resuscitation therapy is beneficial for patients with sepsis and septic shock, shortening the length of ICU stay, reducing the duration of vasopressor use, and reducing the ICU mortality rate of patients with sepsis and septic shock. However, large, multi-center, prospective randomized controlled trials are needed to further verify these results.

\section{Trial registration}

ChiCTR, ChiCTR1900026084. Registered 20 September 2019 - prospective registered, http://www.chictr.org.cn/edit.aspx?pid=40910\&htm=4

\section{Background}


Sepsis is a common disease that is easily complicated by a variety of complications. Approximately 1.7 million cases occur annually in the United States, with more than 270,000 deaths. [1] According to the population-based epidemiological survey by Bin Du and colleagues, the incidence of sepsis in China is $461 / 100000$ of the population. [2] Although intensive medical care has achieved substantial progress, sepsis and septic shock are still the most common causes of death in the intensive care unit. Even if patients with sepsis survive during hospitalization, residual organ dysfunction that requires continued treatment after discharge is common, such as sepsis leading to acute kidney injury followed by chronic renal failure. [3] Over the last 30 years, more than 100 phase II and III clinical trials have been conducted to test novel drugs and therapeutic interventions designed to improve the outcomes of patients with sepsis and septic shock. However, none of these efforts has resulted in a new drug that directly targets the pathophysiological effects of sepsis. Despite the high mortality and morbidity rates, antibiotics and infection source control remain the sole focus of treatment for the disease. [4]

Recently, Paul Marik et al. [5] published papers in Chest describing the application of metabolic resuscitation therapy in patients with sepsis and septic shock. In this study, the treatment of patients with sepsis and septic shock with a combination of vitamin C, vitamin B1 and hydrocortisone reduced the mortality rate, shortened the time of vascular active drug use and reduced the levels of procalcitonin (PCT). It may represent a new completely approach for treating sepsis and septic shock. Because the trial had a retrospective, before-and-after design, we conducted this prospective randomized controlled trial to further clarify the effectiveness, possible pathophysiological principles, and potential applications of metabolic resuscitation therapy in patients with sepsis and septic shock.

\section{Methods And Materials}

\subsection{Clinical data}

We enrolled patients with sepsis and septic shock from Sep. 2019 to Mar. 2020. A tabular form was used to collect the following information: age, gender, use of mechanical ventilation, arterial lactate level at admission and $6 \mathrm{hr}$ after admission (GEM3000 blood gas analyzer), PCT level at admission and $72 \mathrm{hr}$ after admission (using the VIDAS BRAHMS PCT assay, BioMerieux, Inc., Marcy I 'Etoile, France), use of vasopressin, APACHE II score.

\subsection{Design}

The study was a prospective, randomized controlled trial. This trial was approved by the ethics committee of the Lanzhou University Second Hospital. The ethics committee approval number is 2018-043. The Chinese Clinical Trial Registry number is ChiCTR1900026084. Randomization was performed with the use of a centralized computer generated assignment sequence at the first day in the ICU. And block randomization were used as a method of random grouping. The intervention treatment was initiated on the first day. All the included patients or their families provided written informed consent (if the patient was unable to sign the form due to the use of sedatives, coma, etc., informed consent was provided by the patient's immediate family). 
1.3 Inclusion and exclusion criteria:

1. Consistent with the diagnostic criteria for sepsis 3.0, patients were diagnosed with sepsis when a definite or suspicious infective focus was present and the qSOFA score was greater than or equal to 2 (score standard: systolic pressure $\leq 100 \mathrm{~mm} \mathrm{Hg}, 1$ point; GCS $\leq 13,1$ point; and respiratory rate greater than 22 breaths per minute, 1 point) and there was evidence of organ failure. And

2. PCT $>2 \mathrm{ng} / \mathrm{ml}$.

Exclusion criteria:

1. age $<18$ years;

2. pregnant women;

3. liver failure; and

4. G6PD deficiency.

\subsection{Methods}

We calculated that we would need to enroll 140 patients for the trial to have $80 \%$ power to show ICU mortality, at a two-sided alpha level of $5 \%$.Patients with sepsis and septic shock who were admitted to the ICU from Sep. 2019 to Mar. 2020 were prospectively enrolled. According to the computerized random sequence table, the patients were randomly divided into the experimental group and the control group (the random sequence was placed in a sealed envelope with no light transmission). All patients with sepsis were included in the research cluster of initial therapy (measurement of the arterial lactate level, adequate fluid resuscitation, empiric broad-spectrum antibiotics, vasoactive agents (noradrenaline was preferred, when the dosage of norepinephrine $>20 \mathrm{mcg} / \mathrm{min}$, then it was combined with $0.03 \mathrm{U} / \mathrm{min}$ vasopressin), and maintain the mean arterial pressure (MAP) $₫ 65 \mathrm{mmHg}$ ). $₫$ See Appendix $1 \otimes T$ The control group received standard care only, and the experimental group received standard care and metabolic resuscitation, including vitamin $\mathrm{C}$ (1.5 $\mathrm{g}$ in an intravenous infusion q6h for 3 days), vitamin B1 (200 $\mathrm{mg}$ in an intramuscular injection $\mathrm{q} 12 \mathrm{~h}$ for 3 days), and hydrocortisone (50 $\mathrm{mg}$ in an intravenous infusion $\mathrm{q} 6 \mathrm{~h}$ for 7 days).

\subsection{Outcomes}

1. ICU length of stay (ICU LOS);

2. Duration of vasoactive drugs (time from the initial use the vasoactive drugs to the time of withdrawal, the preferred injection of norepinephrine was used to maintain MAP > $65 \mathrm{mmHg}$; when the dosage of norepinephrine was greater than $20 \mathrm{mcg} / \mathrm{min}$, then $0.03 \mathrm{U} / \mathrm{min}$ vasopressin was added);

3. $72 \mathrm{hr}$ PCT clearance rate (initial PCT minus PCT at 72 hours, divided by the initial PCT and multiplied by 100) at admission; 
4. $6 \mathrm{hr}$ arterial lactate clearance rate (initial arterial lactate level minus the arterial lactate level at 6 hours after admission, divided by the initial arterial lactate value and multiplied by 100); and

5. ICU mortality rate.

Statistical analysis

SPSS 21.0 software (SPSS Inc., Chicago, IL, USA) was used for the statistical analysis of the data. If the measured data displayed a normal distribution, the means \pm standard deviations $(x \pm s)$ were reported. The $T$ test was used to compare the data between the two groups, and the q-q normal probability graph was used for the normality test. If the measured data did not display a normal distribution, the median (M) and interquartile ranges ( $\mathrm{QL}, \mathrm{Qu})$ were used to present the measured data. Count data were reported as $\mathrm{n}$ (\%). Pearson's test was used to compare the dichotomous data between the two groups, and the MannWhitney $\mathrm{U}$ test was used for the ordered classification of multiple groups. All tests were two-sided, and $\mathrm{P}$ $<0.05$ was defined as a statistically significant difference.

\section{Results}

According to the inclusion and exclusion criteria, 136 patients with sepsis and septic shock were included in the study and randomly divided into two groups: 68 in the experimental group and 68 in the control group. $96 \%$ of patients in the experimental group and $94 \%$ in the control group completed standard care, respectively. And $100 \%$ of patients in the experimental group and $99.6 \%$ in the control group received adequate initial antibiotic treatment (The type of antibiotics should be used according to the location of infection and the infection indicators), respectively. The baseline data collected from the two groups were comparable, $P>0.05$, as shown in Table 1 . Among the 136 patients with sepsis and septic shock included in this study, pneumonia was the main cause, as it occurred in $33(49 \%)$ and $41(60 \%)$ patients in the experimental group and control group, respectively. Gram-negative bacilli were the main pathogenic bacteria $(112 / 136,82.4 \%)$ and there were 2 negative cultures in the experimental group and 3 negative cultures in the control group. The ICU length of stay for the experimental group and control group was 9 $(7-12)$ and $11(9-14)$ days, respectively, $P=0.002$. The duration of vasoactive drug use (hours) was 20.8 \pm 9.9 and $46.7 \pm 12.8$, respectively, $P=0.000$. The $6 \mathrm{hr}$ lactate clearance rate was $66.2 \%(55.5,76.7)$ and $30.1 \%(15.6,50)$ in the experimental group and control group, respectively, $\mathrm{P}=0.000$. The $72 \mathrm{hr}$ PCT clearance rate was $70 \%(57.5,80.3)$ and $40.7 \%(24.8,52.2)$, respectively, $P=0.000$. The ICU mortality rate was $8.8 \%(6 / 68)$ and $15 \%(15 / 68)$ in the experimental group and control group, $P=0.033$, as shown in Table 2. Univariate and multivariate logistic regression analyses were used to determine the risk factors for outcomes in patients treated with metabolic resuscitation. Age, sex, mechanical ventilation, SOFA score, metabolic resuscitation, adequacy of initial resuscitation and initial antibiotic therapy were chosen as factors for ICU mortality, as shown in Table 3.

\section{Discussion}


The concept of sepsis dates back to Hippocrates and was initially described as "corrupt". With the development of basic medicine, such as physiology and anatomy, sepsis was gradually recognized as "poison in the blood". However, with the development of pathophysiology and modern medicine, a growing number of experts believe that sepsis is actually organ dysfunction caused by the maladjustment of the host to an infection. For centuries, the concept, criteria, treatment, and prognosis of sepsis have been debated. The Surviving Sepsis Campaign (SSC), which is co-sponsored by the American Society of Critical Care Medicine and the European Society of Critical Care Medicine, has updated three editions of guidelines on sepsis, including a series of recommendations on the concept of and diagnostic criteria for sepsis. However, most of these recommendations have not been verified by large, multicenter, randomized controlled trials, and thus the guidelines have been questioned and criticized by a large number of experts and scholars [6-8]. The reason is the lack of a thorough understanding of the pathophysiological process of sepsis. Therefore, although numerous clinical trials have been conducted on sepsis and septic shock, they have not provided any positive guidance for the clinical treatment of sepsis and septic shock, and the questions arising from the delisting of activated protein $\mathrm{C}$ to $6 \mathrm{~h}$ and $3 \mathrm{~h}$ bundles in the clinical diagnosis and treatment of sepsis have not been fundamentally resolved.

However, the concept of "metabolic resuscitation" that was recently proposed by Paul Marik et al. [5]appears to represent a new approach for the treatment of sepsis. The authors provided a detailed description of the roles and mechanisms of vitamin C, vitamin B1, and hydrocortisone (also known as vitamin S) in sepsis.

Thiamine (vitamin B1) is a water-soluble vitamin that is a key component of cellular metabolism. Its phosphorylated form, thiamine pyrophosphate, functions as a cofactor of pyruvate dehydrogenase, converting pyruvate to acetyl-CoA and entering the tricarboxylic acid cycle (6-8). When the thiamine content is insufficient, pyruvate is not converted to acetyl-CoA, causing an impairment in the aerobic chain and a shift to the anaerobic pathway, leading to elevated serum lactate levels.[9-11]

Similar to thiamine, ascorbic acid (vitamin C) is also a water-soluble vitamin that is essential for many processes in the body. Ascorbic acid, an antioxidant, is an electron donor that directly scavenges free radicals by inhibiting the NADPH oxidase (NOX) pathway, preventing the formation of new free radicals and assisting with the circulation of other antioxidants.[12-14] The antioxidant activity of ascorbic acid reduces endothelial permeability, improves microvascular and macrovascular functions, and reduces cell apoptosis under pathological conditions.[13, 15, 16] Ascorbic acid is essential for the endogenous production of vasopressin and may have an important role in maintaining the reactivity of vasopressin. [17-19]

Many large-scale randomized trials have evaluated the additional benefits of corticosteroids as part of the treatment for septic shock. According to these studies, corticosteroids generally improve a variety of clinical outcomes of septic shock (e.g., time to shock reversal and number of ventilation-free days) but have different outcomes for mortality. [20-22] The routine use of hydrocortisone in patients with septic shock remains a topic of debate.[23, 24] However, the biological basis for the inclusion of hydrocortisone 
in drug combinations is based on the potential synergistic effects of ascorbic acid and hydrocortisone. [25] The absorption of ascorbic acid in cells is mediated by the sodium-vitamin C transporter (SVCT2), whose expression is downregulated in response to inflammation. The use of glucocorticoids has been shown to increase the expression of transporters.[26, 27] Marik and colleagues [5] designed and published a retrospective, before-and-after study in 2017 describing metabolic resuscitation in patients with sepsis. The authors concluded that metabolic resuscitation reduces the hospital mortality rate, shortens the duration of vasoactive drug use and reduces the PCT levels. However, due to the retrospective, before-and-after design and the small sample size (94 cases), the effect of metabolic resuscitation on reducing the mortality rate may vary. To our knowledge, at least, 3 RCTs had been published up to now regarding the same intervention in septic patients. The ORANGES trial [28] was a randomized, double-blinded, placebo-controlled study. The results suggested that the combination of intravenous ascorbic acid, thiamine, and hydrocortisone significantly reduced the time to resolution of shock. However, there were no significant differences between study arms in ICU and hospital mortality, ICU and hospital LOS, ventilator free days, and PCT-C. The HYVCTTSSS trial [29] revealed that the metabolic resuscitation did not reduce mortality compared with placebo in septic patients. And the VITAMINS trail, [30]which enrolled 216 patients fulfilling the Sepsis-3 definition of septic shock, showed that treatment with intravenous vitamin $\mathrm{C}$, hydrocortisone, and thiamine does not lead to a more rapid resolution of septic shock compared with intravenous hydrocortisone alone. Until now, the role of metabolic resuscitation in patients with sepsis and septic shock is still controversial. Therefore, our trial was designed as a prospective randomized controlled trial to further clarify the role of metabolic resuscitation in patients with sepsis and septic shock. Our study showed that the mortality rate of both groups was lower than previous studies (the VITAMINS trail was $19.6 \%$, the HYVCTTSSS trial was $27.5 \%$ and the ORANGES trial was $9 \%$ ), there may be several reasons: First, the included patients had an APACHE II score of less than 15 , so it was possible to conclude that the mortality rate was lower than in previous studies. Second, 6 hours lactate clearance rate and 72 hours PCT clearance rate were significantly higher than that of control group, in the univariate analysis showed higher 6 hours lactate clearance rate and 72 hours PCT clearance rate were related with adequacy of initial antibiotic therapy and adequacy of initial resuscitation, and the $P$ value is less than 0.05 .So that the timely initiation of antibiotic therapy and fluid resuscitation may also have contributed to the lower mortality rate than in previous studies. In the original plan, vitamin B1 was injected intravenously, but because the intravenous route was not mentioned in the specification for vitamin B1 in China, intramuscular injection was used instead.

\section{Limitations}

Our study still has several limitations. First, it is a single-center study that included a homogeneous patient population. Second, we did not measure the concentration of vitamin C. Finally, our study was designed solely according to the protocol reported by Marik. Although the results showed that metabolic resuscitation may reduce the mortality associated with sepsis and septic shock in patients, the results should be interpreted with caution, and the mechanism by which metabolic resuscitation therapy treats sepsis requires further study in the future. 


\section{Conclusions}

Metabolic resuscitation therapy is beneficial for patients with sepsis and septic shock because it shortens the stay in the ICU, reduces the duration of vasoactive drug use, and reduces the ICU mortality rate of patients with sepsis and septic shock. However, further validation in large, multicenter, prospective randomized controlled trials is still needed.

\section{Declarations}

\section{Ethics approval and consent to participate}

The Ethics Committee of LanZhou University Second Hospital approved this study, and the number was 2018-043. Written informed consent was obtained from individual or guardian participants.

\section{Consent for publication}

Not applicable.

\section{Availability of data and materials}

All data generated or analysed during this study are included in this published article.

\section{Competing interests}

All authors declare that there are no competing interests.

\section{Funding}

Not applicable.

\section{Authors' contributions}

Conducted the study: YC, FF.

Collected all data: ML, H-hY, W-wY.

Did the statistical analysis: YC, FF.

\section{Acknowledgements}

We would like to thank AJE (www.aje.cn) for English language editing.

\section{Authors` information}

${ }^{1}$ Intensive Care Unit 2 of Lanzhou University Second Hospital; 
2 Intensive Care Unit of Peoples` Hospital of Linxia State;

\section{References}

[1] Rhee C, Dantes R, Epstein L, Murphy DJ, Seymour CW, Iwashyna TJ, et al. Incidence and Trends of Sepsis in US Hospitals Using Clinical vs Claims Data, 2009-2014. Jama. 2017;318:1241-9.

[2] Zhou J, Tian H, Du X, Xi X, An Y, Duan M, et al. Population-Based Epidemiology of Sepsis in a Subdistrict of Beijing. Critical care medicine.1.

[3] Rhodes A, Evans LE, Alhazzani W, Levy MM, Dellinger RP. Surviving Sepsis Campaign: International Guidelines for Management of Sepsis and Septic Shock. Critical care medicine. 2017;45:1.

[4] Hollenberg S, Ahrens T, Astiz M, Chalfin D, Dasta J, Heard S, et al. Practice parameters for hemodynamic support of sepsis in adult patients in sepsis. Task Force of the American College of Critical Care Medicine, Society of Critical Care Medicine. 1999;27:639.

[5] Marik PE, Khangoora V, Rivera R, Hooper MH, Catravas J. Hydrocortisone, Vitamin C, and Thiamine for the Treatment of Severe Sepsis and Septic Shock: A Retrospective Before-After Study. Chest. 2017;151:1229-38.

[6] Marik PE. SEP-1: The Lactate Myth and Other Fairytales. Critical care medicine. 2018;46:1689-90.

[7] Marik PE. Fluid Responsiveness and the Six Guiding Principles of Fluid Resuscitation. Critical care medicine. 2016;44:1920-2.

[8] Marik PE. Self-plagiarism: the perspective of a convicted plagiarist! European journal of clinical investigation. 2015;45:883-7.

[9] Mallat J, Lemyze M, Thevenin D. Do not forget to give thiamine to your septic shock patient! Journal of thoracic disease. 2016;8:1062-6.

[10] Gioda CR, de Oliveira Barreto T, Primola-Gomes TN, de Lima DC, Campos PP, Capettini Ldos S, et al. Cardiac oxidative stress is involved in heart failure induced by thiamine deprivation in rats. American journal of physiology Heart and circulatory physiology. 2010;298:H2039-45.

[11] Pacal L, Tomandl J, Svojanovsky J, Krusova D, Stepankova S, Rehorova J, et al. Role of thiamine status and genetic variability in transketolase and other pentose phosphate cycle enzymes in the progression of diabetic nephropathy. Nephrology, dialysis, transplantation : official publication of the European Dialysis and Transplant Association - European Renal Association. 2011;26:1229-36.

[12] Mortensen A, Lykkesfeldt J. Does vitamin C enhance nitric oxide bioavailability in a tetrahydrobiopterin-dependent manner? In vitro, in vivo and clinical studies. Nitric oxide : biology and chemistry. 2014;36:51-7. 
[13] Oudemans-van Straaten HM, Spoelstra-de Man AM, de Waard MC. Vitamin C revisited. Critical care (London, England). 2014;18:460.

[14] Wu F, Tyml K, Wilson JX. iNOS expression requires NADPH oxidase-dependent redox signaling in microvascular endothelial cells. Journal of cellular physiology. 2008;217:207-14.

[15] Amrein K, Oudemans-van Straaten HM, Berger MM. Vitamin therapy in critically ill patients: focus on thiamine, vitamin C, and vitamin D. Intensive care medicine. 2018;44:1940-4.

[16] Kc S, Carcamo JM, Golde DW. Vitamin C enters mitochondria via facilitative glucose transporter 1 (Glut1) and confers mitochondrial protection against oxidative injury. FASEB journal : official publication of the Federation of American Societies for Experimental Biology. 2005;19:1657-67.

[17] Victor VV, Guayerbas N, Puerto M, Medina S, De la Fuente M. Ascorbic acid modulates in vitro the function of macrophages from mice with endotoxic shock. Immunopharmacology. 2000;46:89-101.

[18] Armour J, Tyml K, Lidington D, Wilson JX. Ascorbate prevents microvascular dysfunction in the skeletal muscle of the septic rat. Journal of applied physiology. 2001;90:795-803.

[19] Carcamo JM, Pedraza A, Borquez-Ojeda O, Golde DW. Vitamin C suppresses TNF alpha-induced NF kappa B activation by inhibiting I kappa B alpha phosphorylation. Biochemistry. 2002;41:12995-3002.

[20] Venkatesh B, Finfer S, Cohen J, Rajbhandari D, Arabi Y, Bellomo R, et al. Adjunctive Glucocorticoid Therapy in Patients with Septic Shock. The New England journal of medicine. 2018;378:797-808.

[21] Annane D, Renault A, Brun-Buisson C, Megarbane B, Quenot JP, Siami S, et al. Hydrocortisone plus Fludrocortisone for Adults with Septic Shock. The New England journal of medicine. 2018;378:809-18.

[22] Sprung CL, Annane D, Keh D, Moreno R, Singer M, Freivogel K, et al. Hydrocortisone therapy for patients with septic shock. The New England journal of medicine. 2008;358:111-24.

[23] Rochwerg B, Oczkowski SJ, Siemieniuk RAC, Agoritsas T, Belley-Cote E, D'Aragon F, et al. Corticosteroids in Sepsis: An Updated Systematic Review and Meta-Analysis. Critical care medicine. 2018;46:1411-20.

[24] Rygard SL, Butler E, Granholm A, Moller MH, Cohen J, Finfer S, et al. Low-dose corticosteroids for adult patients with septic shock: a systematic review with meta-analysis and trial sequential analysis. Intensive care medicine. 2018;44:1003-16.

[25] Okamoto K, Tanaka H, Makino Y, Makino I. Restoration of the glucocorticoid receptor function by the phosphodiester compound of vitamins $\mathrm{C}$ and E, EPC-K1 (L-ascorbic acid 2-[3,4-dihydro-2,5,7,8-tetramethyl2-(4,8,12-trimethyltridecyl)-2H-1-benzopyran-6 -yl hydrogen phosphate] potassium salt), via a redoxdependent mechanism. Biochemical pharmacology. 1998;56:79-86. 
[26] Fujita I, Hirano J, Itoh N, Nakanishi T, Tanaka K. Dexamethasone induces sodium-dependant vitamin C transporter in a mouse osteoblastic cell line MC3T3-E1. The British journal of nutrition. 2001;86:145-9.

[27] Barabutis N, Khangoora V, Marik PE, Catravas JD. Hydrocortisone and Ascorbic Acid Synergistically Prevent and Repair Lipopolysaccharide-Induced Pulmonary Endothelial Barrier Dysfunction. Chest. 2017;152:954-62.

[28] Iglesias J, Vassallo AV, Patel VV, Sullivan JB, Cavanaugh J, Elbaga Y. Outcomes of Metabolic Resuscitation Using Ascorbic Acid, Thiamine, and Glucocorticoids in the Early Treatment of Sepsis: The ORANGES Trial. Chest. 2020;158:164-73.

[29] Chang P, Liao Y, Guan J, Guo Y, Zhao M, Hu J, et al. Combined Treatment With Hydrocortisone, Vitamin C, and Thiamine for Sepsis and Septic Shock: A Randomized Controlled Trial. Chest. 2020;158:174-82.

[30] Fujii T, Luethi N, Young PJ, Frei DR, Eastwood GM, French CJ, et al. Effect of Vitamin C, Hydrocortisone, and Thiamine vs Hydrocortisone Alone on Time Alive and Free of Vasopressor Support Among Patients With Septic Shock: The VITAMINS Randomized Clinical Trial. Jama. 2020;323:423-31.

\section{Tables}

Table 1 Baseline Information 


\begin{tabular}{|llll|}
\hline & Experimental group $(\mathrm{n}=68)$ & Control group $(\mathrm{n}=68)$ & P value \\
\hline Age & $57.2 \pm 14.4$ & $56.7 \pm 15.1$ & 0.84 \\
\hline Sex (male) & $40(59 \%)$ & $42(62 \%)$ & 0.727 \\
\hline Infection Source & & & 0.881 \\
\hline Pneumonia & $33(49 \%)$ & $41(60 \%)$ & \\
\hline BSI & $7(10 \%)$ & $9(13 \%)$ & \\
\hline Urosepsis & $6(9 \%)$ & $4(6 \%)$ & \\
\hline GI & $8(12 \%)$ & $6(9 \%)$ & 0.997 \\
\hline Other & $14(20 \%)$ & $8(12 \%)$ & 0.091 \\
\hline Comorbidities & & & 0.085 \\
\hline Diabetes & $24(35 \%)$ & $27(40 \%)$ & 0.254 \\
\hline Heart Failure & $20(29 \%)$ & $15(22 \%)$ & 0.756 \\
\hline CoPD & $19(28 \%)$ & $21(31 \%)$ & 0.632 \\
\hline Hypertension & $5(8 \%)$ & $4(7 \%)$ & 0.882 \\
\hline Mechanical Ventilation & $37(54 \%)$ & $41(60 \%)$ & 0.943 \\
\hline Use of Vasoactive Drugs & $49(72 \%)$ & $30(44 \%)$ & 0.069 \\
\hline Arterial Lactate Level (mmol/l) & $3.8 \pm 0.99$ & $3.8 \pm 2.4$ & 0.399 \\
\hline Procalcitonin Level (ng/ml) & $35(28-100)$ & $63(19-47)$ & \\
\hline Day 1 SOFA Score & $9.1 \pm 3.2$ & $67(99.6 \%)$ & \\
\hline APACHE II score & $11.9 \pm 5.6$ & & \\
\hline Adequacy of initial resuscitation & $65(96 \%)$ & 68.6 & \\
\hline Adequacy of antibiotic therapy & $68(100 \%)$ & & \\
\hline
\end{tabular}

BSI: Blood Stream Infection

Table 2 Outcome and treatment variables 


\begin{tabular}{|c|c|c|c|}
\hline & $\begin{array}{l}\text { Experimental group } \\
(n=68)\end{array}$ & $\begin{array}{l}\text { Control group } \\
(\mathrm{n}=68)\end{array}$ & $P$ value \\
\hline ICU LOS (days) & $9(7-12)$ & $11(9-14)$ & $P=0.002$ \\
\hline $\begin{array}{l}\text { Duration of vasoactive drug use } \\
\text { (hours) }\end{array}$ & $20.8 \pm 9.9$ & $46.7 \pm 12.8$ & $\begin{array}{r}P Q \\
0.001\end{array}$ \\
\hline Lactate Clearance Rate (6 hr) & $66.2 \%(55.5,76.7)$ & $30.1 \%(15.6,50)$ & $\begin{array}{r}P Q \\
0.001\end{array}$ \\
\hline Procalcitonin Clearance Rate (72 hr) & $70 \%(57.5,80.3)$ & $40.7 \%(24.8,52.2)$ & $\begin{aligned} P Q \\
0.001\end{aligned}$ \\
\hline ICU Mortality Rate (\%) & $8.8 \%(6 / 68)$ & $15 \%(15 / 68)$ & $P=0.033$ \\
\hline
\end{tabular}

Table 3 Multivariate analysis for the impact of factors on ICU mortality

\begin{tabular}{|c|c|c|}
\hline Variable & Odds ratio $(95 \% \mathrm{Cl})$ & $P$ Value \\
\hline Age & $0.486 \rrbracket 0.093$ to $1.546 \rrbracket$ & 0.664 \\
\hline Sex & $0.889 \llbracket 0.211$ to $2.276 \rrbracket$ & 0.769 \\
\hline SOFA score & $1.182 \varangle 0.933$ to $1.497 \rrbracket$ & 0.483 \\
\hline Adequacy of initial resuscitation & $0.26 \rrbracket 0.21$ to $1.75 \rrbracket$ & 0.042 \\
\hline Adequacy of initial antibiotic therapy & $1.007 \rrbracket 0.111$ to $9.102 \rrbracket$ & 0.248 \\
\hline Mechanical ventilation & $0.74 \llbracket 0.276$ to $1.987 \rrbracket$ & 0.281 \\
\hline APACHE II score & $1.932 \varangle 0.819$ to $1.052 \rrbracket$ & 0.087 \\
\hline Use of Vasoactive Drugs & $1.22 \varangle 0.94$ to $1.53 \rrbracket$ & 0.051 \\
\hline Arterial Lactate Level & $0.044 \llbracket 0.023$ to $0.069 \rrbracket$ & 0.000 \\
\hline Procalcitonin Level & $0.91 \rrbracket 0.54$ to $1.587 \rrbracket$ & 0.300 \\
\hline
\end{tabular}

